\title{
Using multi-criteria analysis for undertaking vulnerability assessments of flood susceptible communities
}

\author{
J. Perdikaris \\ PhD Candidate Water Resources Engineering, \\ University of Guelph, Canada
}

\begin{abstract}
This paper presents a methodology for assessing the vulnerability of communities, specifically as it relates to flooding using multi-criteria analysis and a risk management approach. Risk is assessed as a combination of threat (expressed as the probability that a given flood will occur); vulnerability (expressed as the probability that a given vulnerability will succeed, given that the flood will occur), and consequence (expressed as some measure of loss, such as dollar cost, resources loss, programmatic impact, etc.). Many factors contribute to a communities' vulnerability with respect to flooding. These include its population, built environment, and wealth, which are increasingly concentrated in a small number of highly vulnerable areas that are susceptible to flooding.

The total risk is assessed as a combination of the risks associated with a specific flooding scenario and its impact on a susceptible community. Risk is reduced by countermeasures. However, the cost of countermeasures (relative to the potential risks) is applied when arriving at an all encompassing risk management strategy.

The ongoing phenomenon of climate change is altering our capacity to effectively manage the risks associated with flooding. Moreover, it is affecting North America in particularly distinct ways. In the past 10 years, Canada in particular has faced the greatest increases in average annual temperatures of any country, and a commensurate rise in severe weather-related natural disasters has been observed across North America. Associated with these shifts has been an increased occurrence of flooding, further exacerbating the situation and making communities more vulnerable to its effects.
\end{abstract}

Keywords: flooding, risk management, vulnerability and coping capacity. 


\section{Introduction}

The process of assessing the vulnerability of an area to flood hazard entails determining the following: Who is vulnerable? What is vulnerable? Where is it located? In determining the vulnerability of a population with respect to flooding, indicators need to be established to identify areas with multiple criteria for assessing vulnerability, which require specific consideration for the population at risk.

There are many aspects that can be incorporated into a vulnerability index, such as monetary vulnerability, social vulnerability, environmental vulnerability, economic vulnerability and critical infrastructure vulnerability. This paper will focus on a methodology used for estimating the direct and indirect costs associated with damages from the flood waters, social vulnerability and environmental vulnerability as it pertains to environmentally sensitive areas located within the Flood Damage Centres (FDCs); areas that have identified as being susceptible to flood waters.

\section{Flood disasters and their impacts}

A disaster occurs only if an extreme event such as a flood strikes a vulnerable population. However, what is meant by vulnerability needs to be specified.

In the traditional meaning, a disaster is a state, in which a population, population group or individual is not able to cope or is not able to overcome the adverse effects of an extreme event, without outside help. When examining extreme event, without outside help.

When examining extreme events, such as flooding; although the primary cause for a flood is extreme rainfall or snowmelt, or combination of both; the impact or magnitude of a flood is determined by human influences. For example,

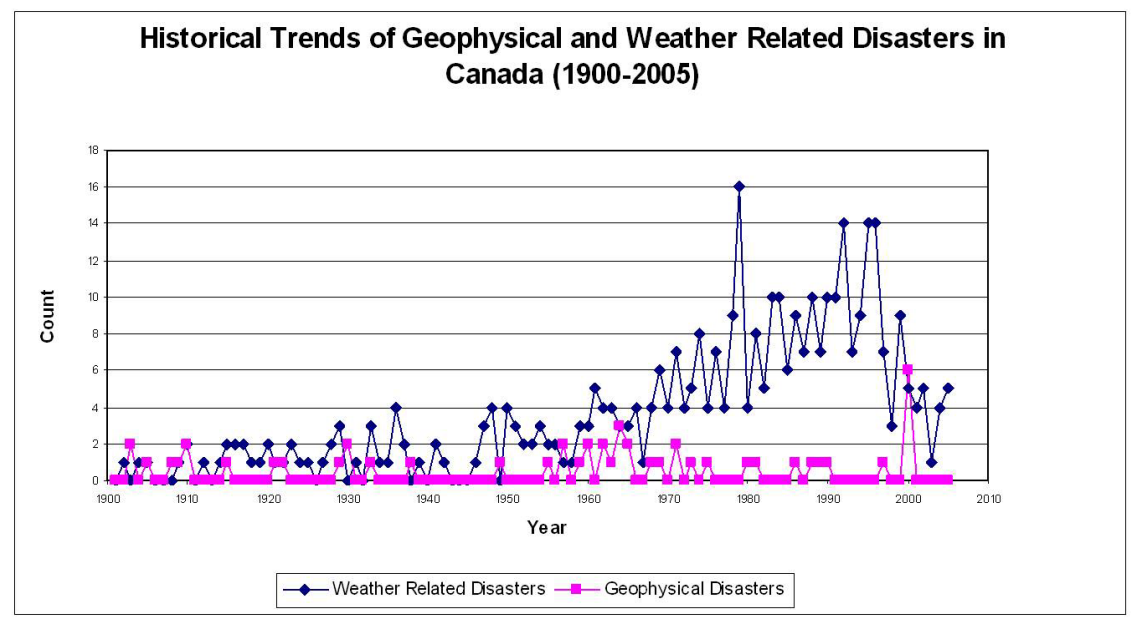

Figure 1: Historical trends of geophysical and weather related disasters in Canada (1900-2005; public safety Canada, disasters database). 
the definition of disaster as a state where people at risk can no longer help themselves; conforms to the modern view of a disaster as a social event, where people at risk are vulnerable to an extreme event because of their social conditions. According to this view, disaster management is not only a technical task, but is also a social task.

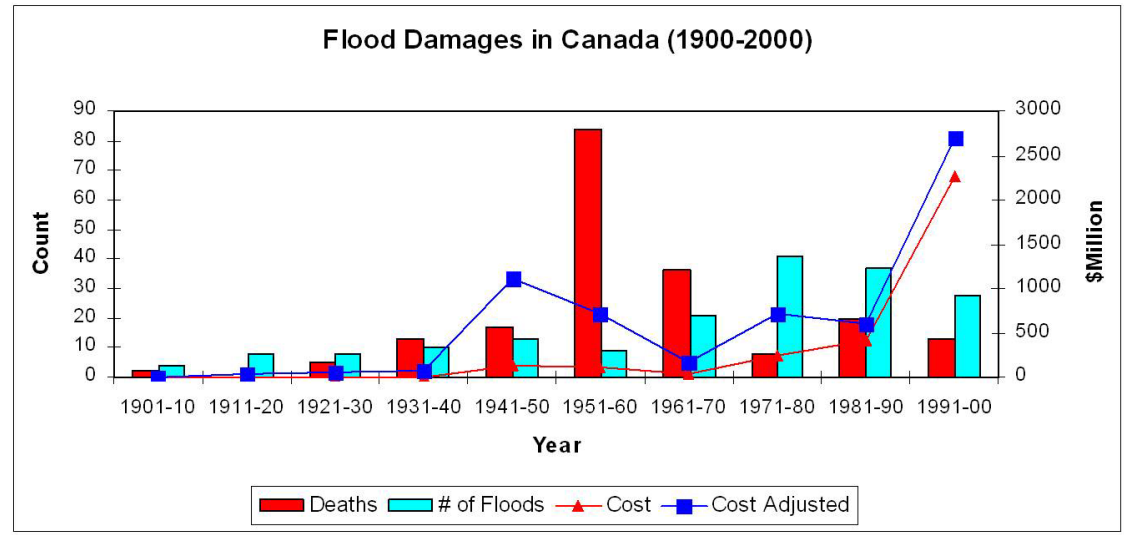

Figure 2: Flood damages in Canada (1900-2000; public safety Canada, disasters database).

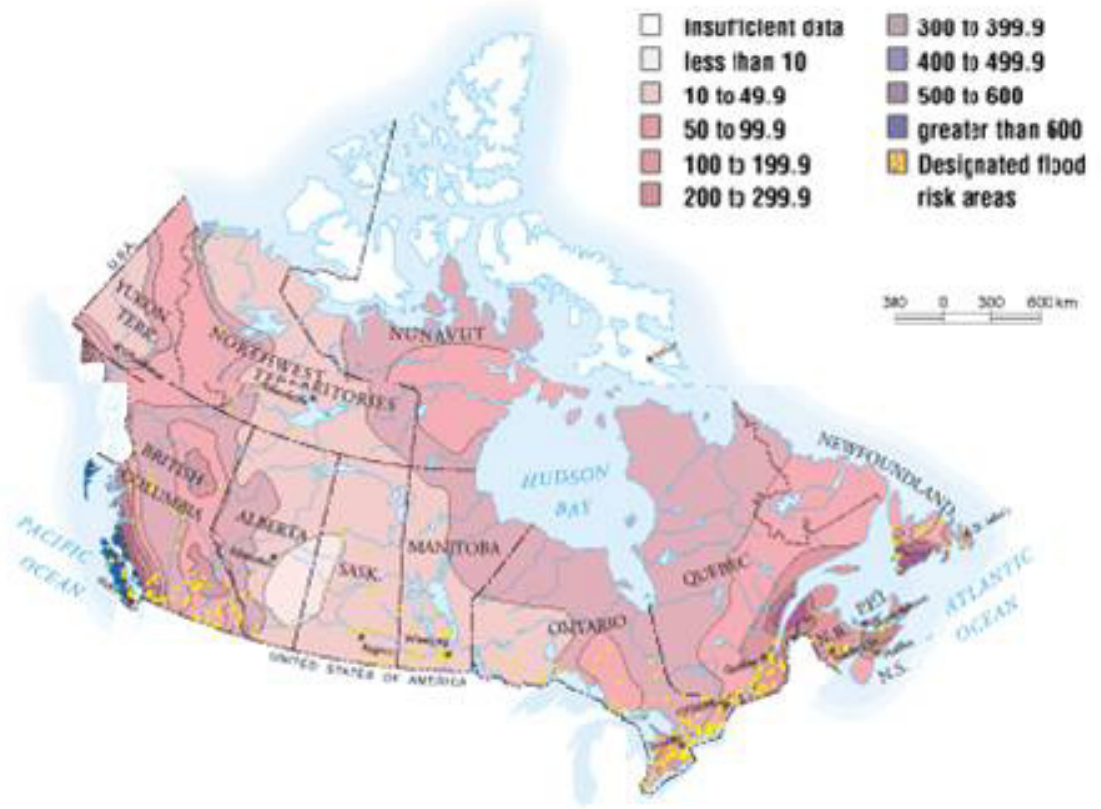

Figure 3: Designated flood risk areas within Canada (public safety Canada, 2009). 
People are vulnerable to water related hazards, specifically, floods. Between 1960 to present the number of weather related disasters has been increasing (Figure 1). In addition, the cost associated with flood damages has also been increasing (Figure 2). For example, in 1997 the Red River floods in Manitoba resulted in 4 deaths and 25,000 people being evacuated. The total cost in damages or losses was estimated to be approximately $\$ 1.0$ billion. The previous year the Saguenay flood resulted in 10 deaths and 15,000 people being evacuated. The total cost in damages was estimated to be $\$ 1.5$ billion. Most areas which have been designated as flood risk are those with high population densities (Figure 3). According to Figure 3, areas that have designated as "flood risk areas" are those areas that have high population densities, conforming to the modern view of a disaster as a social event.

\section{Vulnerability}

Vulnerability is a function of exposure, sensitivity, and adaptive capacity. Differential vulnerabilities exist across different systems based on inherent response abilities. Both humans and natural systems are affected. Climate Change is an additional and multi-pronged stressor. A challenging task is to define factors (indices) that quantify the impact on human vulnerability of flood disasters, and obtain a quantitative basis for decision making. The United Nations defines this factor as the "index of human security."

\subsection{Indices of vulnerability and human security}

An indicator, comprising a single data point (or a single variable) or an output value from a set of data (aggregation of variables), is a quantity that describes a system or process such that it has significance beyond the face value of its components. It aims to communicate information on the system or process. The dominant criterion behind an indicator's specification is scientific and/or engineering knowledge and judgement (World Water Development Report, 2003).

An index is a mathematical aggregation of variables or indicators, often across different measurement units so that the result is dimensionless. The purpose of an index is to provide compact and targeted information for management and policy development and decisions. The problem of combining the individual components is overcome by scaling and weighting processes, which are not absolute, but reflect social preferences (Plate [3]).

\subsection{Types of vulnerability}

There five (5) types of vulnerability: monetary vulnerability, social vulnerability, environmental vulnerability, economic vulnerability and critical infrastructure vulnerability. Economic vulnerability is the costs incurred or money lost through loss of production, distribution and consumption. Critical infrastructure vulnerability is a measure of the effect of damage to critical infrastructure, including telecommunications, electricity, drinking water and sewer services. 
Because the latter require significant amounts of data to process in order to quantify the vulnerability index, the focus will be on the monetary, social and environmental vulnerabilities.

\subsubsection{Monetary vulnerability}

The monetary vulnerability is defined as the cost of damages or the cost of reestablishing previous conditions after a disaster. The monetary vulnerability has well-established methodologies associated with it and has been studied fairly extensively. Generally, flood damage curves are developed from the costs associated with previous events (i.e. FIA curves in the US; or Flood Damage Assessment Curves, Ontario Ministry of Natural Resources) or from extensive interviews with home owners to identify the type and condition of housing and what would be damaged inside the house at different flood depths (Schuster et al. [10]; McBean et al. [7]). The former approach is likely to underestimate damages as a result of people not accounting for personal time spent cleaning up after a flood, but overestimate damages based on insurance claims from individuals who overstate the damages incurred. The latter approach is labour and time- intensive for data collection and requires people to hypothesise as to their behaviour during a flood event, which may be very different under the stress of the actual situation. Another approach is to use lifestyle classes, rather than residence classes to calculate depth - damage costs (Schuster et al. [10]; Experian [5]).

The damages due to flooding are usually divided into direct and indirect damages. The direct damages comprise the tangible damages to property that result from contact with or submersion under water, while the indirect damages are as a result of interruption to physical and economic linkages, such as the interruption of traffic flow patterns as a result of a flooded road, and loss of income or business profits (Schuster et al. [10]; Messner and Meyer [8]). Other approaches have been developed, however they have been deemed too data and computationally intensive to undertake and are beyond the scope of this paper. Another intangible flood cost which is not examined herein is anxiety. It has been argued that anxiety affects productivity, which in turn affects Gross National Product (GNP) (Schuster et al. [10]; Lekuthai and Vongvisessomjai [6]).

\subsubsection{Social vulnerability}

Social Vulnerability is defined as the condition of persons-at-risk, their integration into the community and their access to vital services such as, stresses and hazards that negatively impact social cohesion. Dwyer et al. [4] and Schuster et al. [10] undertook detailed questionnaires to identify and quantify factors that contribute to vulnerability to a natural hazard. Fifteen rules were identified that lead to characterization of vulnerability. These rules were applied using synthetic population estimation in order to generate vulnerability maps for different scenarios. The scenarios were based on severity of injury and direct damages to homes given a damage event. Age, income, gender, employment, residence type, household type, tenure type, health insurance, house insurance, car ownership, 
disability, English language skills, debt/savings were identified as playing a role in household vulnerability.

Complete destruction of a residence is the great equalizer, making everyone vulnerable. Apart from this, the rules demonstrated that it is a combination of attributes which make a person/household vulnerable. The relative importance of individual indicators was such that injuries, followed by damage to the house, were at the top. House insurance, income and tenure type were next, followed by age, while household type, health insurance, residence type, gender and disability were the lowest scoring indicators (Schuster et. al. [10]; Dwyer et al. [4]). Figure 4 demonstrates the social vulnerability model used in the study by Dwyer et al. [4]. De Almeida et al. [3] depict social vulnerability using a different approach (Figure 5).

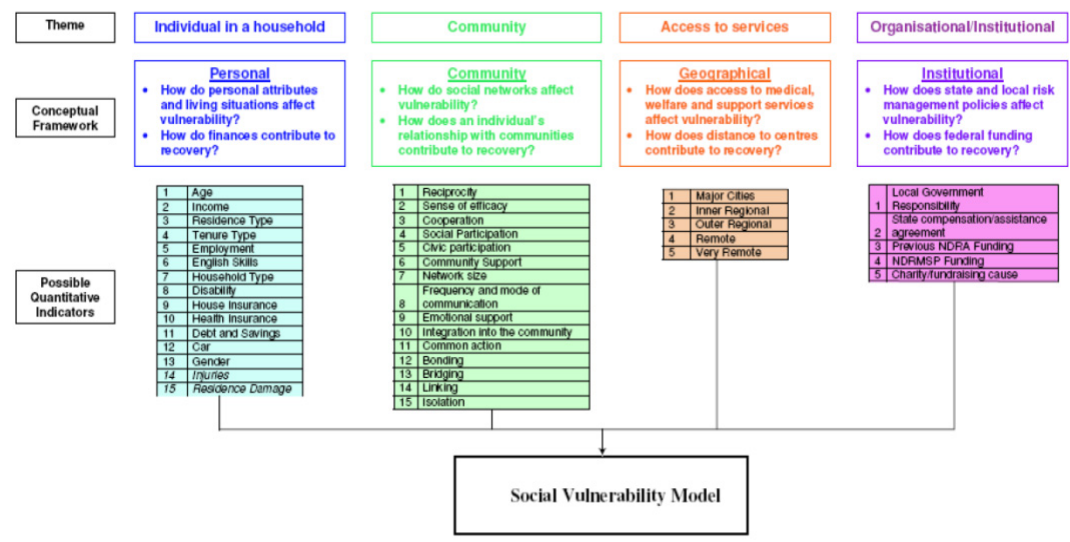

Figure 4: $\quad$ Factors associated with social vulnerability (Dwyer et al. [4]).

Vulnerability indices may assume a variety of formats. Relevant indices can be aggregated to form a single index or separate indices can be developed which represent different vulnerabilities. Additionally, an index can be constructed through geographical groupings, or by profiling individual geographic entities (Schuster et. al. [10]; Adger et al. [1]). The Social Flood Vulnerability Index (SFVI) (Schuster et. al. [10]; Tapsell et al. [13]) is a composite additive index which incorporates both social characteristics and financial-deprivation indicators. The study (Tapsell et al. [13] and Schuster et. al. [10]), based on the analysis of other studies undertaken in the UK identifies age and financial status as common key indicators. The Townsend Index (Townsend et al. [14]) was used to identify financially deprived households. Another financial deprivation index is the Carstairs Index (Carstairs and Morris [2]) which incorporates car ownership, household crowding, unemployment and a social class variable.

The SFVI consists of the Townsend Index, weighted at 0.25 , a 'long-term sick' variable (residents with limiting, long term illness as a percentage of the population), single parents and, the elderly (age 75 and over). The final index is calculated by summing the standardized Z-scores. 


\section{Vulnerability type Dimension Indicators}

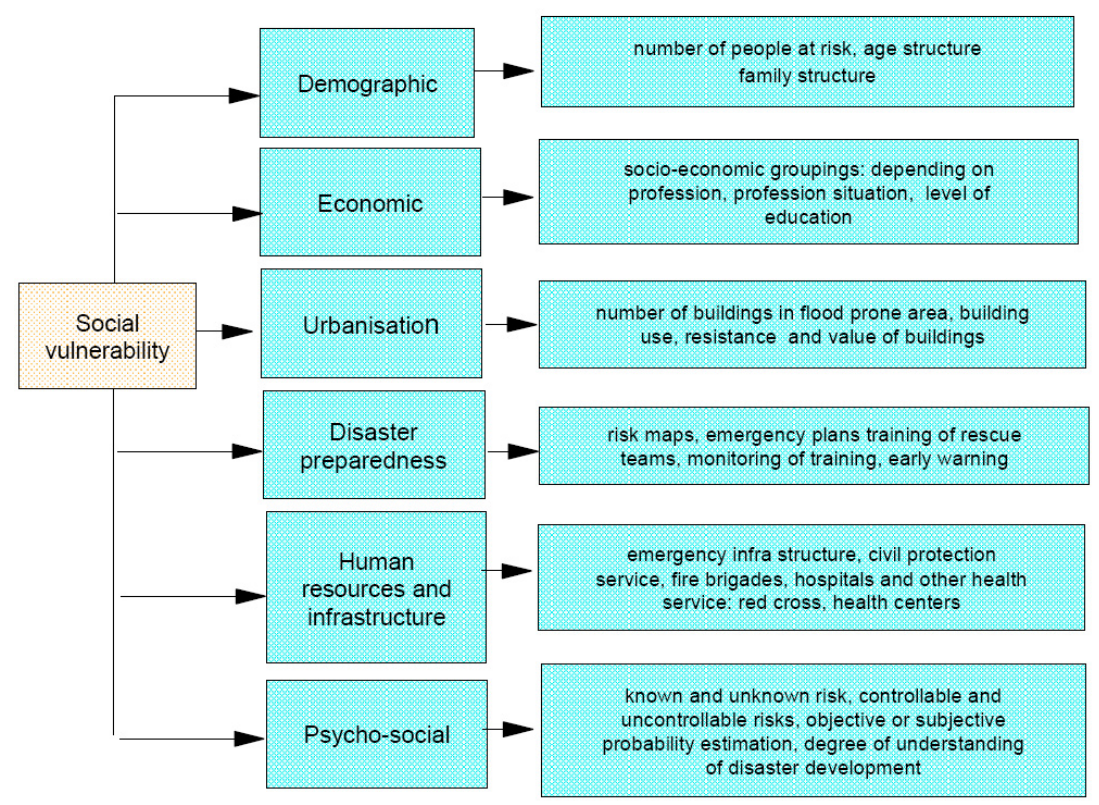

Figure 5: Dimensions of and indicators for vulnerability (after De Almeida, et al. [3]).

Rygel et al. [9] and Schuster et al. [10] used poverty, gender, race and ethnicity, age, and disabilities to develop a vulnerability index. Composite indices can have inherent problems. When averaging factors, the importance of an individual factor is reduced and an overall index may classify an area as 'not vulnerable', while an individual factor may indicate 'extreme vulnerability'. When weighting factors, it is difficult to identify the weights to be applied. The assignments can be subjective, relying on expert judgement, rather than quantitative. Additionally, weights cannot account for variations in relative importance of factors over space and time. In order to avoid the problems associated with averaging or weighting, a method was developed which undertook principle component analyses on 57 variables. The top 3 components that were used in the development of the index can be described as poverty, immigrants and old age / disabilities. To avoid averaging the three parameters for each geographic area, Pareto rankings were used to classify the regions according to the significance of all three components.

St. Bernard [11] and Schuster et al. [10] include life expectancy at birth, security, social order and governance indicators (i.e. indictable crimes per 100,000 population), resource allocation indicators (i.e. the proportion of children belonging to the poorest quintiles, and communications architecture (i.e. computer literacy rate). 
As indicated in the above, health is an important aspect of vulnerability. After a flood event, individual health can be affected by the stress and trauma associated with loss of possessions. Enteric illnesses can be prevalent, given exposure to contaminated water. Cramped conditions in evacuation shelters increase transmission of these illnesses. General damp conditions and the growth of mould on affected surfaces can exacerbate chest conditions, including asthma. It can be difficult to obtain appointments for, or travel to, physician's offices and this can escalate underlying conditions (Schuster et al. [10]; Tapsell et al. [13]).

\subsubsection{Environmental vulnerability}

Environmental vulnerability is the damage to the environment as it affects the health of natural ecosystems. Areas that have been designated as environmentally sensitive, such wetlands and fishery spawning areas are particularly vulnerable to flooding. The latter could also result in economic damages due to loss of fisheries stock, when a community is reliant on fishing as an economic livelihood for commercial harvesting purposes.

Climate change is an unforeseen stressor and needs to be incorporated into the overall vulnerability index quantification method. Given the level of uncertainty associated with climate change, several future climate scenarios need to be examined in order to quantify their risk and impact. For example, two commonly used climate scenarios in Southern Ontario include a "hotter-wetter" and "hotterdryer" scenarios. By examining different climate scenarios, uncertainty bands can be established for predicting future floods. This approach could also be used to establish uncertainty bands for other indicators such as demographics based on population forecasts.

\section{Quantification of vulnerability}

Vulnerability is defined as the total resources needed for meeting the demands of a natural or extreme event (Plate [3]):

$$
V=V_{s}+V_{e}
$$

In equation $1 \mathrm{~V}$ is defined as the total Vulnerability and $\mathrm{V}_{\mathrm{s}}$ is the amount needed to maintain the social standard or the basic needs vulnerability and $V_{e}$ is the consequence of an event. The values of $\mathrm{V}_{\mathrm{e}}$ and $\mathrm{V}_{\mathrm{s}}$ are computed using the equations 2 and 3 (Plate [3]):

$$
\begin{aligned}
& V_{e}=\gamma_{11} V_{e E}+\gamma_{21} V_{e S}+\gamma_{31} V_{e R} \\
& V_{s}=\gamma_{12} V_{s E}+\gamma_{22} V_{s S}+\gamma_{32} V_{s R}
\end{aligned}
$$

The subscripts E, S and R represent the economic, societal and environmental dimensions. Each vulnerability index $V_{e} E, S, R$ or $V_{S E, S, R}$ is represented by the sum of the indicators for each dimension multiplied by a weighting factor $\gamma$, as illustrated in equation 4 (Schuster et. al., 2007). The subscript $\mathrm{m}$ represents the type of vulnerability, "consequence of an event" (e) or "basic needs" (s). The subscript $\mathrm{N}$ represents the dimension, $\mathrm{E}, \mathrm{S}$ or $\mathrm{R}$, and the subscript (i) is the indicator for a specific dimension. 


$$
\begin{gathered}
V_{m N}=\sum_{i=1}^{i}\left(V_{m i} * \gamma_{m i}\right) \\
V_{m i}=\frac{\text { population }- \text { group }-i}{\text { TotalPopulation }}
\end{gathered}
$$

The value of $\mathrm{V}_{\mathrm{mi}}$ as illustrated in equation 5 is the percentage of the population group for indicator (i) of the total population for the flood inundated area. The weighting factors $(\gamma)$ are based on three (3) scenarios: The first weighting scenarios $(\gamma)_{0}$, does not apply weights to any of the categories. The second weighting factors $(\gamma)_{1}$ are comparatively derived, by identifying the importance of key variables over others, as derived from the literature. And the third weighting factors $(\gamma)_{2}$ are derived from a questionnaire or survey to professionals or "flood experts", which asks individuals to rank each variable. The scores are than summed and represented as a ratio of the average score. The differences in numerical values of the vulnerability indices between the three (3) weighting schemes, provides a sensitivity analysis of vulnerability scores, as well as identifying flood vulnerable areas (Schuster et al. [10]).

Consequently we can quantify the coping capacity by means of the monetary value, MA as illustrated by equation 6 (Plate [3]).

$$
M A=V_{\text {critical }}-V_{s}
$$

In monetary terms, $\mathrm{V}_{\text {critical }}$, is the total funds available, and MA is the value of the coping capacity. The long term indices are based on the expected values of $\mathrm{V}_{\text {critical }}, \mathrm{V}_{\mathrm{s}}$ and $\mathrm{V}_{\mathrm{e}}$. In addition, the long term index is equivalent to the total risk or total risk index (RI) divided by the coping capacity (MA).

\section{Adaptation strategy}

For improving our own security with respect to flooding, appropriate mitigation measures must be developed or improved, based on the recognition of changes both in vulnerability and in resistance. There are three (3) distinct ways of flood mitigation: reduce the vulnerability, increase resistance and reduce the impact. The approach then becomes an optimization problem, which can be solved through a risk management approach. For example, a risk is defined as a hazard, multiplied by the elements of exposure to that hazard and the vulnerability. Therefore, a hazard assessment leads to a vulnerability assessment which in turn leads to a risk assessment. This operational model is an ongoing process in which the risk management plan and mitigation plan are developed and/or refined to meet the societal objectives and capabilities of the community, both technical and administrative based on a sound risk assessment. This would to an increase in our coping capacity and our resistance and thereby reducing our vulnerability.

Furthermore, the climate risk assessment approach along with the forecasts of the numerical indices and uncertainty bands for population dynamics need to be incorporated into the vulnerability assessments to suitable adaptation measures. 
This needs to be further enhanced by integrating the climate risk screening results and population growth statistics into the flood mitigation program design and implementation. By incorporating these variables into our planning process can we begin to predict long term changes within our communities, and thereby assist us in developing a range of credible adaptation options.

\section{References}

[1] Adger WN, Brooks N, Bentham G, Agnew M and Eriksen S. 2004. New indicators of vulnerability and adaptive capacity. Tyndall Centre Technical Report 7 Tyndall Centre for Climate Change Research Norwich, UK.

[2] Carstairs V and Morris R. 1991. Deprivation and Health in Scotland. Aberdeen University Press, Aberdeen.

[3] De Almeida A, Matias Ramos C, Santos MA, Viseu T (eds). 2003. Dam Break Flood Risk Management in Portugal. Laboratorico Nacional de Engenharia Civil, Lisbon, Portugal, as found in Plate, 2004.

[4] Dwyer A, Zoppou C, Nielson O, Day S and Roberts S. 2004. Quantifying Social Vulnerability: A methodology for identifying those at risk to natural hazards. Geoscience Australia Record 2004/14.

[5] Experian. 2000. Available from: http://www.climatechange.govt.nz/ resources/reports/economic-impacts-extreme-events-jul04/html/page $3 . h t m l$ (Accessed January 2010).

[6] Lekuthai A and Vongvisessomjai S. 2001. Intangible flood damage quantification. Water Resources Management 15:343-362.

[7] McBean EA, Gorrie J, Fortin M, Ding J and Moulton R. 1988. Flood depthdamage curves by interview survey. Journal of Water Resources Planning and Management 114(6):613-33 18.

[8] Messner F and Meyer V. 2005. Flood Damage, Vulnerability and Risk Perception - Challenges for Flood Damage Research. UFZ Discussion Papers, Dept. of Economics 13/2005.

[9] Rygel L, O'Sullivan D and Yarnal B. 2005. A Method for Constructing a Social Vulnerability Index. Submitted to Mitigation and Adaptation Strategies for Global Change 21 January 2005.

[10] Schuster, C.J., Murray, S., and McBean, E.A. 2007. Vulnerability Characterization, Mapping, and Assessment: A Study of Flooding Scenarios for the Credit River Watershed. Report Submitted to Credit Valley Conservation.

[11] St. Bernard G. 2003. Towards the Construction of a Social Vulnerability Index -Theoretical and Methodological Considerations. Journal of Social and Economic Studies, The University of the West Indies, Mona Jamaica Submitted 2003.

[12] Tapsell, S. M. \& Tunstall, S. M. 2001 The health and social effects of the June 2000 flooding in the northeast region. Report to the Environment Agency. Flood Hazard Research Centre, Middlesex University, Enfield. 
[13] Tapsell SM, Penning-Roswell EC, Tunstall SM and Wilson TL. 2002. Vulnerability to flooding: health and social dimensions. Phil. Trans. R. Soc. London A 360:1511-25.

[14] Townsend P, Phillimore P and Beattie A. 1988. Health and deprivation: inequality and the North. London: Croom Helm. 